\title{
Variabilidade climática sazonal e anual da chuva e veranicos no Estado do Paraná
}

\author{
Rosandro Boligon Minuzzi ${ }^{1}$; Paulo Henrique Caramori ${ }^{2}$
}

\section{RESUMO}

As mudanças climáticas na escala regional são muito divergentes e nem sempre acompanham a tendência global. Metodologias estatísticas foram utilizadas para analisar o comportamento sazonal e anual da chuva e de veranicos, em 21 estações hidrológicas localizadas no Estado do Paraná. Os resultados obtidos pelo Teste de Homogeneidade Normal Padrão não indicam pontos de mudanças estatisticamente significativos, no comportamento climático da quantidade de chuva. Entretanto, a Análise de Regressão e o teste de Kendall mostram que, nas últimas décadas, a quantidade de chuva anual tem aumentado, principalmente na primavera, entre 16 e $42 \mathrm{~mm} /$ década, na metade leste do Paraná. A mesma tendência foi observada na intensidade dos eventos extremos diários de chuva, mas os municípios e períodos sazonais com esta característica não seguem rigorosamente as tendências apresentadas na quantidade de chuva. O número de tendências significativas (predominantemente negativas) é menor para os veranicos, com duração de 5 a 10 dias, com quantidade acumulada inferior a $10 \mathrm{~mm}$, porém, mais perceptível na primavera e na escala anual.

Palavras-chave: tendência climática, ponto de descontinuidade, chuva extrema.

\begin{abstract}
Seasonal and annual climatic variability of rainfall and dry spells in the state of Paraná, Brazil

Climatic changes on a regional scale are very divergent and not always accompany the global tendency. Statistical methods were used to analyze the patterns of seasonal and annual rainfall and dry spells in 21 hydrological stations located in the State of Paraná, southern Brazil. The results obtained by the Standard Normal Homogeneity Test indicated that there were no significant changes in the climatic patterns of rainfall. But the regression analysis and Kendall test showed that, in recent decades, the amount of annual rainfall has increased from 16 to $42 \mathrm{~mm} / \mathrm{decade}$ in the eastern half of Paraná state, especially in the spring. The same trend was observed in the intensity of extreme daily rainfall events, but municipalities and seasons with this feature did not strictly follow the trends presented in the amount of rainfall. The number of significant trends (predominantly negative) is less for the dry spells lasting 5 to 10 days with accumulated rainfall $<10 \mathrm{~mm}$, but more noticeable in the spring and on an annual scale.
\end{abstract}

Key words: Climate trends, extreme rain, point of discontinuity. 


\section{INTRODUÇÃO}

Dentro do atual contexto de aquecimento global, são esperados eventos de chuvas mais intensos e mais frequentes, como resultado do maior conteúdo de água na atmosfera, favorecido pela maior instabilidade termodinâmica (Kunkel, 2003). Seguindo este raciocínio, o quarto relatório do IPCC mostra que houve aumento de ocorrência de chuvas intensas em muitas regiões, em latitudes médias, durante o período de 1951 a 2003. Estas ocorrências eram esperadas, em razão das mudanças na quantidade total de chuvas (IPCC, 2007). Porém, atenta-se que, na escala regional, essas mudanças são muito divergentes e nem sempre acompanham a tendência global.

Apesar da grande ressalva que se faz, atualmente, quanto à inclusão do fator antropogênico na variabilidade temporal e espacial de elementos meteorológicos, há os fatores naturais, resultantes da interação oceano-atmosfera, que resultam em flutuações interanual e decadal. Feidas et al. (2007) analisaram a tendência anual e sazonal da chuva na Grécia e a relação da variabilidade da chuva, com três índices de circulação atmosférica. Os autores encontraram uma tendência de decréscimo na escala anual, determinada pelo clima observado no inverno, e a Oscilação do Atlântico Norte, como o índice mais correlacionado com a variabilidade da chuva.

As projeções climáticas futuras são feitas com base em tendências observadas no passado. A precisão dos resultados depende da quantidade e da qualidade dos dados registrados. Diante das projeções constantemente divulgadas quanto às mudanças no clima e seus possíveis impactos no ecossistema, tornam-se necessárias informações e ferramentas cada vez mais precisas, visando a aprimorar e atualizar a tomada de decisões. Diante destas considerações, o objetivo deste estudo foi analisar o comportamento climático sazonal e anual da chuva e veranicos no Estado do Paraná.

\section{MATERIAL E MÉTODOS}

Foram utilizados dados diários de chuva de estações hidrológicas localizadas no Estado do Paraná (Figura 1) e pertencentes à Agência Nacional de Águas (ANA). Na Tabela 1, consta o ano de início das séries de chuva de cada estação, que se estendem até 2006.

As lacunas de observações foram preenchidas com dados de estações hidrológicas vizinhas, valendo-se do método de regressão linear como indicador de condições pluviométricas semelhantes, a 5\%, e homogeneidade suficiente para se efetuar o preenchimento. O teste não paramétrico denominado 'run test', ou teste de sequência, foi aplicado para avaliar a homogeneidade das séries meteorológicas. Recomendado pela Organização Meteorológica Mundial (OMM), em sua Nota Técnica, nº 81 (Thom,
1966), o referido teste consiste na contagem do número de ocorrências de valores observados da série temporal, situados abaixo e acima da mediana, testando o resultado da contagem mediante uso de uma tabela de distribuição.

Dois métodos foram utilizados para testar a tendência da altura pluviométrica sazonal (verão, outono, inverno e primavera), anual, dos veranicos e dos eventos extremos diários, a saber, a Análise de Regressão e teste de Kendall (Kendall, 1938). AAnálise de Regressão foi utilizada para indicar alterações climáticas, por meio da significância do coeficiente angular de uma reta ajustada aos dados. O teste consiste em determinar o intervalo de confiança deste coeficiente, sendo que, se este intervalo de confiança não incluir o valor zero, a tendência é significativa. O coeficiente de Kendall é um teste não paramétrico e considera a diferença entre a probabilidade de que os dados observados de duas variáveis estejam na mesma ordem, contra a probabilidade de que os mesmos dados estejam em ordens diferentes, para as referidas variáveis. Um valor positivo do coeficiente de Kendall indica uma tendência de aumento, enquanto um valor negativo indica uma tendência de decréscimo, desde que significativos, de 10 ou $5 \%$, obtidos pelo p-valor. Ambos os testes foram aplicados para três períodos, sendo um para toda a série e, os demais, em intervalos divididos pelo ponto de descontinuidade identificado pelo Teste de Homogeneidade Normal Padrão (THNP). Proposto por Alexandersson (1986), o THNP testa um desvio na média $(\bar{Y})$ e estabelece como hipótese nula que a variável mensal $\mathbf{Y}_{\mathbf{i}}$ (i é o ano de 1 a n) a ser testada é independente e identicamente distribuída. Assim, o teste $\mathbf{T}(\mathbf{k})$ compara a média dos primeiros $\mathbf{k}$ anos de registros mensais com os últimos n-k anos:

$$
T(k)=k \bar{z}_{1}^{2}+(n-k) \bar{z}_{2}^{2} \quad \mathrm{k}=1, \ldots, \mathrm{n}
$$

em que:

$$
\bar{z}_{1}=\frac{1}{k} \sum_{i=1}^{k} \frac{\left(Y_{i}-\bar{Y}\right)}{s} \quad \text { e } \quad \bar{z}_{2}=\frac{1}{n-k} \sum_{i=k+1}^{n} \frac{\left(Y_{i}-Y\right)}{s}
$$

Se o ponto de mudança está localizado no ano K, então $\mathbf{T}(\mathbf{k})$ atinge um máximo próximo ao ano $\mathbf{k}=\mathbf{K}$. O teste estatístico $\mathbf{T}_{\mathbf{0}}$ é definido como:

$$
T_{0}=\max _{1 \leq k<n} T(k)
$$

A hipótese nula será rejeitada se $\mathbf{T}_{\mathbf{0}}$ estiver acima de um determinado nível, que é dependente do tamanho da amostra. Os valores críticos, a 5\%, para o teste estatístico de $\mathbf{T}_{\mathbf{0}}$, adotados neste estudo, foram definidos por Alexandersson \& Moberg (1997).

Os extremos diários de chuva foram considerados como os valores sazonais acima do percentil 0,95. Os meses seguintes foram definidos como representativos de 
cada um dos períodos sazonais: de dezembro a fevereiro (verão), de março a maio (outono), de junho a agosto (inverno) e de setembro a novembro (primavera).

Para a análise de veranicos, considerou-se como dia seco (sem chuva), aquele com quantidade acumulada inferior a 1 $\mathrm{mm}$, pois uma lâmina de chuva inferior a essa geralmente não infiltra no solo. A análise de tendência foi realizada na escala sazonal e anual, para as seguintes situações:

- Número de dias secos (inferior a $1 \mathrm{~mm}$ );

- Sequência de 5 dias com total de chuva inferior a $1 \mathrm{~mm}$; - Sequência de 5 dias com total de chuva inferior a 10 mm;
- Sequência de 10 dias com total de chuva inferior a $10 \mathrm{~mm}$.

A média anual do atual período, considerado pela OMM como 'normal climatológica' (1961-1990), foi comparada com a média do período de 1991 a 2006 por meio da estatística:

$$
t_{n_{1}+n_{2}-2}=\frac{x_{1}-x_{2}}{S_{p} \sqrt{\frac{1}{n_{1}}+\frac{1}{n_{2}}}}
$$

em que:

$\mathrm{x}_{1}$ e $\mathrm{x}_{2}=$ média da chuva anual do período de 1961-1990 e 1991-2006, respectivamente;

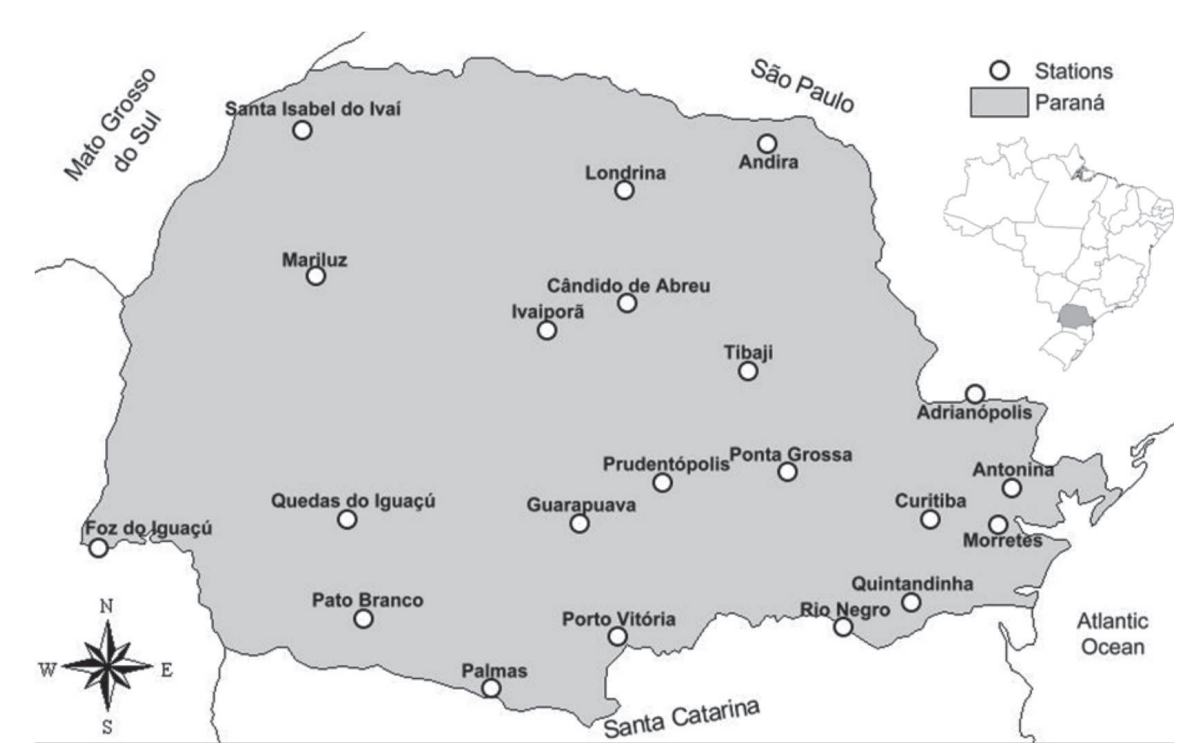

Figura 1. Localização geográfica das estações hidrológicas utilizadas no estudo e localizadas no Estado do Paraná.

Tabela 1. Código, coordenadas e início da série dos municípios onde localizam-se as estações utilizadas no estudo

\begin{tabular}{lcccc}
\hline Municípios & Código & Latitude $\left(^{\circ}\right)$ & Longitude $\left(^{\circ}\right)$ & Início da série \\
\hline Adrianópolis & 02449000 & $-24,65$ & $-48,98$ & 1945 \\
Andirá & 02350002 & $-23,08$ & $-50,28$ & 1946 \\
Antonina & 02548003 & $-25,23$ & $-48,75$ & 1947 \\
Cândido de Abreu & 02451003 & $-24,08$ & $-51,15$ & 1957 \\
Curitiba & 02549006 & $-25,43$ & $-49,26$ & 1890 \\
Foz do Iguaçu & 02554001 & $-25,61$ & $-54,46$ & 1945 \\
Guarapuava & 02551000 & $-25,45$ & $-51,45$ & 1955 \\
Ivaiporã & 02451014 & $-24,25$ & $-51,65$ & 1956 \\
Morretes & 02548000 & $-25,46$ & $-48,83$ & 1958 \\
Pato Branco & 02652009 & $-26,05$ & $-52,80$ & 1946 \\
Ponta Grossa & 02550003 & $-25,13$ & $-50,15$ & 1946 \\
Porto Vitória & 02651004 & $-26,16$ & $-51,21$ & 1946 \\
Prudentópolis & 02550000 & $-25,20$ & $-50,93$ & 1955 \\
Quedas do Iguaçu & 02552000 & $-25,43$ & $-52,90$ & 1945 \\
Quitandinha & 02549003 & $-25,95$ & $-49,38$ & 1925 \\
Rio Negro & 02649021 & $-26,10$ & $-49,80$ & 1958 \\
Sant a Isabel do Ivaí & 02353001 & $-23,00$ & $-53,18$ & 1945 \\
Tibaji & 02450002 & $-24,50$ & $-50,40$ & 1955 \\
Londrina & 02351010 & $-23,37$ & $-51,17$ & 1955 \\
Palmas & 02652010 & $-26,48$ & $-52,00$ & 1958 \\
Mariluz & 02353002 & $-23,91$ & $-53,10$ & \\
\hline
\end{tabular}


$\mathrm{n}_{1}$ e $\mathrm{n}_{2}=$ número de dados do período de 1961-1990 e 1991-2006, respectivamente;

$\mathrm{S}_{\mathrm{p}}=$ média ponderada das variâncias amostrais $\mathrm{S}_{1}^{2} \mathrm{e}$ $\mathrm{S}_{2}^{2}$, definida como:

$$
S_{p}=\sqrt{\frac{\left(n_{1}-1\right) S_{1}^{2}+\left(n_{2}-1\right) S_{2}^{2}}{n_{1}+n_{2}-2}}
$$

Neste estudo, foram testadas as seguintes hipóteses para as variâncias da quantidade de chuva anual do período de 1961-1990 e 1991-2006, utilizando-se o teste $F$ (equação 4), como $F_{\mathrm{v1,v2,1- \alpha}}$ :

$$
\begin{aligned}
& H_{o}:=\left(S_{1}^{2}+S_{2}^{2}\right) \\
& H_{1}:=\left(S_{1}^{2}+S_{2}^{2}\right) \\
& F=\frac{\max \left(S_{1}^{2}, S_{2}^{2}\right)}{\min \left(S_{1}^{2}, S_{2}^{2}\right)}
\end{aligned}
$$

\section{RESULTADOS E DISCUSSÃO}

A Tabela 2 mostra que houve, aproximadamente, $20 \%$ de séries consideradas estatisticamente como 'não homogêneas' (NH). Dentre as 21 estações hidrológicas utilizadas no estudo, o verão (outono) foi o período com a maior (menor) ocorrência de séries 'não homogêneas' e a estação de Ponta Grossa a única a ter mais do que duas situações de períodos sazonais 'não homogêneos'.

As informações da Tabela 3 mostram que houve poucas ocorrências significativas de pontos de descontinuidade, nas séries sazonais e anual de chuvas. Na análise sazonal, esta significância estatística (5 ou 1\%) foi observada apenas uma vez, no verão e no inverno, e em duas situações na primavera, sendo, estas, encontradas no Li- toral do estado e no início da década de 1990. Apesar dos resultados predominantemente não significativos, chama a atenção o elevado número de pontos de descontinuidade, concentrados entre 1992 e 1996, durante o verão (8 casos) e a primavera (14 casos), e entre 1982 e 1984, durante o outono (12 casos). Isto defronta com os resultados encontrados por Obregón \& Nobre (2003), que verificaram mudança no comportamento da chuva no Brasil (exceto no nordeste do Brasil), em meados da década de 1970. Destacam que este comportamento esteve relacionado, principalmente, com a temperatura da superfície do oceano Pacífico.

Desta forma, os resultados de pontos de descontinuidade não sugerem que as quantidades de chuva sazonal ou anual no Paraná estejam sendo influenciadas, significativamente, por algum ciclo climático perceptível no período analisado (entre 50 e 60 anos, em sua maioria). Neste contexto, as séries de dados pluviométricos das estações hidrológicas de Curitiba e Rio Negro, que abrangem um período de 117 a 81 anos, mostraram, cada uma, três pontos de descontinuidade localizados em meados da década de 1990 e da década de 1970, respectivamente.

Assim, sem a identificação de mudanças significativas no comportamento climático da chuva, desde meados do século XX o Estado do Paraná apresenta uma tendência de aumento na quantidade de chuvas, principalmente, durante o verão e a primavera e, possivelmente, refletindo-se na escala anual (Tabela 4). Enquanto, no verão, as quantidades de chuva aumentaram entre 17 e $37 \mathrm{~mm} /$ década, na primavera, esta tendência foi de 16 a $42 \mathrm{~mm} /$ década. Algumas estações hidrológicas apresentaram esta tendência significativa em ambos os períodos sazonais, como Andirá, Antonina, Guarapuava, Morretes, Prudentópolis,

\begin{tabular}{|c|c|c|c|c|}
\hline Municípios & Verão & Outono & Inverno & Primavera \\
\hline Adrianópolis & $\mathrm{NH}$ & $\mathrm{H}$ & $\mathrm{H}$ & $\mathrm{NH}$ \\
\hline Andira & $\mathrm{NH}$ & $\mathrm{H}$ & $\mathrm{H}$ & $\mathrm{H}$ \\
\hline Antonina & $\mathrm{H}$ & $\mathrm{H}$ & $\mathrm{H}$ & $\mathrm{H}$ \\
\hline Cândido de Abreu & NH & $\mathrm{H}$ & $\mathrm{NH}$ & $\mathrm{H}$ \\
\hline Curitiba & $\mathrm{H}$ & $\mathrm{H}$ & $\mathrm{NH}$ & $\mathrm{H}$ \\
\hline Foz do Iguaçu & $\mathrm{H}$ & $\mathrm{H}$ & $\mathrm{H}$ & $\mathrm{H}$ \\
\hline Guarapuava & $\mathrm{H}$ & $\mathrm{H}$ & NH & $\mathrm{NH}$ \\
\hline Ivaiporã & $\mathrm{H}$ & $\mathrm{H}$ & $\mathrm{H}$ & $\mathrm{H}$ \\
\hline Morretes & NH & $\mathrm{H}$ & $\mathrm{H}$ & $\mathrm{H}$ \\
\hline Pato Branco & $\mathrm{H}$ & NH & $\mathrm{H}$ & $\mathrm{H}$ \\
\hline Ponta Grossa & $\mathrm{NH}$ & $\mathrm{H}$ & NH & NH \\
\hline Porto Vitória & $\mathrm{H}$ & $\mathrm{H}$ & $\mathrm{H}$ & $\mathrm{H}$ \\
\hline Prudentópolis & $\mathrm{H}$ & $\mathrm{H}$ & $\mathrm{H}$ & $\mathrm{H}$ \\
\hline Quedas do Iguaçu & $\mathrm{H}$ & $\mathrm{H}$ & $\mathrm{H}$ & $\mathrm{H}$ \\
\hline Quitandinha & $\mathrm{H}$ & $\mathrm{H}$ & $\mathrm{H}$ & $\mathrm{H}$ \\
\hline Rio Negro & NH & $\mathrm{H}$ & $\mathrm{H}$ & NH \\
\hline Santa Isabel do Ivaí & $\mathrm{H}$ & $\mathrm{H}$ & $\mathrm{H}$ & $\mathrm{H}$ \\
\hline Tibaji & NH & $\mathrm{H}$ & $\mathrm{H}$ & NH \\
\hline Londrina & $\mathrm{H}$ & $\mathrm{H}$ & NH & $\mathrm{H}$ \\
\hline Palmas & $\mathrm{H}$ & $\mathrm{H}$ & $\mathrm{H}$ & $\mathrm{H}$ \\
\hline Mariluz & $\mathrm{H}$ & $\mathrm{H}$ & $\mathrm{H}$ & $\mathrm{H}$ \\
\hline
\end{tabular}
Rio Negro e Tibaji, independentemente da homogeneidade

Tabela 2. Resultados do teste de sequência (Run test) sazonal para definição das séries de chuva como homogênea (H) ou não homogênea $(\mathrm{NH})$

Rev. Ceres, Viçosa, v. 58, n.5, p. 593-602, set/out, 2011 
ou não de suas séries. Como exemplos, em Antonina e Morretes (Litoral paranaense), desde meados da década de 1940, as quantidades de chuva no verão e na primavera aumentaram em torno de 177 e $226 \mathrm{~mm}$, respectivamente. Os resultados das chuvas são coerentes com os obtidos por Obregón \& Marengo (2007), que, analisando séries históricas de chuvas anuais, nos últimos 50 anos, constataram um aumento no total de chuvas para todo o sul do país, assim como na frequência e intensidade dos eventos extremos. Com os dados de 1928 a 1998, registrados em Urussanga (Litoral Sul de Santa Catarina), Back (2001) também encontrou tendência significativa de aumento na chuva anual durante o trimestre outubro-dezembro.

As estações hidrológicas com maior número de tendências significativas, na primavera e na escala anual, estão concentradas na metade leste do estado, ou seja, em áreas mais próximas ao oceano Atlântico, mas acrescidas

Tabela 3. Pontos de descontinuidade no comportamento pluviométrico sazonal e anual para alguns municípios do Paraná com o ano inicial da análise

\begin{tabular}{|c|c|c|c|c|c|c|}
\hline \multirow{2}{*}{ Municípios } & \multirow{2}{*}{ Início da série } & \multicolumn{5}{|c|}{ Ponto de descontinuidade da chuva } \\
\hline & & Verão & Outono & Inverno & Primavera & Anual \\
\hline Adrianópolis & 1945 & 1998 & 1982 & 1957 & 1999 & 1981 \\
\hline Andira & 1946 & 1988 & 1982 & 2004 & 1986 & 1985 \\
\hline Antonina & 1947 & 1993 & 1996 & 1969 & $1992 *$ & 1992 \\
\hline Cândido de Abreu & 1957 & 2002 & 1982 & $1957 *$ & 1957 & 1957 \\
\hline Curitiba & 1890 & 1994 & 1982 & 2005 & 1992 & 1992 \\
\hline Foz do Iguaçu & 1945 & 1994 & 1982 & 1998 & 1992 & 1988 \\
\hline Guarapuava & 1955 & 1993 & 1982 & 1955 & 1995 & 1991 \\
\hline Ivaiporã & 1956 & 2003 & 2005 & 1957 & 1957 & 1957 \\
\hline Morretes & 1945 & 1992 & 1945 & 1989 & $1992 * *$ & $1991 *$ \\
\hline Pato Branco & 1958 & 2004 & 1982 & 2001 & 1995 & 1978 \\
\hline Ponta Grossa & 1946 & 2002 & 1999 & 2001 & 1995 & 1968 \\
\hline Porto Vitória & 1946 & 1947 & 1998 & 2005 & 1996 & 2005 \\
\hline Prudentópolis & 1946 & $1994 *$ & 1982 & 1988 & 1994 & $1988 *$ \\
\hline Quedas do Iguaçu & 1955 & 2004 & 1982 & 1955 & 1995 & 1983 \\
\hline Quitandinha & 1945 & 1945 & 1945 & 1969 & 1994 & 1968 \\
\hline Rio Negro & 1925 & 1993 & 1982 & 1979 & 1974 & $1974 *$ \\
\hline Santa Isabel do Ivaí & 1958 & 1993 & 1982 & 2004 & 1958 & 1988 \\
\hline Tibaji & 1945 & 1970 & 2005 & 2004 & 1994 & 1970 \\
\hline Londrina & 1955 & 1955 & 1956 & 2004 & 1998 & 2002 \\
\hline Palmas & 1955 & 1957 & 2002 & 1955 & 1993 & 1957 \\
\hline Mariluz & 1958 & 2004 & 1984 & 2000 & 1995 & 1963 \\
\hline
\end{tabular}

** significativo a $5 \%$, significativo a $1 \%$.

Tabela 4. Coeficientes angulares para toda a série da quantidade de chuva sazonal e anual

\begin{tabular}{|c|c|c|c|c|c|}
\hline \multirow[b]{2}{*}{ Municípios } & \multicolumn{5}{|c|}{ Quantidade de chuva (toda a série) } \\
\hline & Verão & Outono & Inverno & Primavera & Anual \\
\hline Adrianópolis & ns & ns & ns & ns & ns \\
\hline Andira & $2,0 * *$ & ns & ns & $1,8 * *$ & $4,8 * *$ \\
\hline Antonina & $2,9 *$ & ns & $1,2 *$ & $2,7 * *$ & $5,1 * *$ \\
\hline Cândido de Abreu & ns & $2,3 *$ & ns & ns & $4,6^{*}$ \\
\hline Curitiba & ns & $0,6^{*}$ & ns & ns & ns \\
\hline Foz do Iguaç u & ns & ns & ns & $2,1 * *$ & $4,1 *$ \\
\hline Guarapuava & $2,8 * *$ & ns & ns & $4,2 * *$ & $7,1 * *$ \\
\hline Ivaiporã & ns & ns & ns & ns & $\mathrm{ns}$ \\
\hline Morretes & $3,7 * *$ & ns & $1,8 * *$ & $3,7 * *$ & $9,5 * *$ \\
\hline Pato Branco & ns & ns & ns & $3,4^{*}$ & $7,6^{*}$ \\
\hline Ponta Grossa & ns & ns & ns & $2,6 * *$ & $4,6^{*}$ \\
\hline Porto Vitória & ns & ns & ns & ns & ns \\
\hline Prudentópolis & $3,3 * *$ & ns & ns & $2,6 * *$ & $7,2 * *$ \\
\hline Quedas do Iguaçu & ns & ns & ns & $3,9 * *$ & ns \\
\hline Quitandinha & ns & ns & $1,3 *$ & $1,8 * *$ & $4,6 * *$ \\
\hline Rio Negro & $1,8 * *$ & $1,4^{*}$ & $1,4 * *$ & $1,6^{* *}$ & $5,9 * *$ \\
\hline Santa Isabel do Ivaí & ns & ns & ns & ns & ns \\
\hline Tibaji & $1,7 *$ & ns & ns & $2,7 * *$ & $5,6 * *$ \\
\hline Londrina & ns & ns & ns & ns & ns \\
\hline Palmas & ns & ns & ns & $4,2 * *$ & ns \\
\hline Mariluz & ns & ns & ns & $2,4^{*}$ & ns \\
\hline
\end{tabular}

ns= não significativo; $* *$ Significativo a $5 \% ; *$ Significativo a $10 \%$. 
de eventuais áreas a sudoeste do Estado. Esta configuração pode estar associada ao aumento das chuvas oriundas da circulação marítima, ou no aumento da frequência de frentes frias, mais atuantes nas áreas próximas ao Atlântico. Analisando a climatologia de frentes frias, de 1979 a 2005, sobre a América do Sul, Cavalcanti \& Kousky (2009) encontraram que, das duas regiões com maior frequência no continente, uma delas é ao longo da costa leste do Brasil, onde, na média, de 5 a 10 frentes frias chegam até $15^{\circ} \mathrm{S}$, a cada ano. Por sua vez, os sistemas meteorológicos podem ser influenciados por modos de variabilidade climática interanual e interdecadal, como o El Niño Oscilação Sul (ENOS) e a Oscilação Decadal do Pacífico (Pacific Decadal Oscillation - PDO), respectivamente. Porém, Re \& Barros (2009) destacam que, para o sudeste da América do Sul, especificamente na região que envolve a parte do sul do Brasil, a tendência positiva encontrada na ocorrência de chuvas fortes de 1959 a 2002, tem grande probabilidade de não ser resultante de variabilidade interanual.

A Figura 2 apresenta o comportamento da chuva anual e na primavera, para duas estações hidrológicas localizadas em Andirá e Prudentópolis, com tendência significativa de aumento. Nesta figura, percebe-se que a partir da década de 1990 houve pouca ou nenhuma quantidade acumulada considerada numa situação de 'seca' (abaixo do quartil 0,25). Alguns estudos, como de Marengo (2004), associam esse comportamento chuvoso, em grande parte do Brasil (principalmente durante os anos 1990), às várias ocorrências do fenômeno climático El Niño (EN) que tem como característica resultar em chuvas acima da média climática nas áreas ao sul do Brasil (Diaz et al., 1998; Diniz et al., 1998; Grimm 2000). Seguindo este raciocínio, Silva Dias (2009) constatou um aumento significativo da temperatura do ar e da chuva anual, na região litorânea de Santa Catarina, no período de 1950 a 2000. Por causa de uma mudança nas anomalias, de negativas para positivas, em meados da década de 1970, os pesquisadores sugeriram que isto pode estar associado com fenômenos de escala planetária, como o ENOS e a PDO.

Na Tabela 5, apresentam-se as tendências dos extremos diários de chuva sazonal. Num contexto geral, há tendência de aumento nesta característica, mas sem a identificação de um período sazonal, predominantemente, com as maiores ocorrências. Essa tendência é condizente com o aumento nas quantidades de chuva. Porém, a mesma associação não é aplicada rigorosamente na análise por estações hidrológicas. Como exemplos, os municípios de Adrianópolis e Rio Negro possuem tendência de aumen-
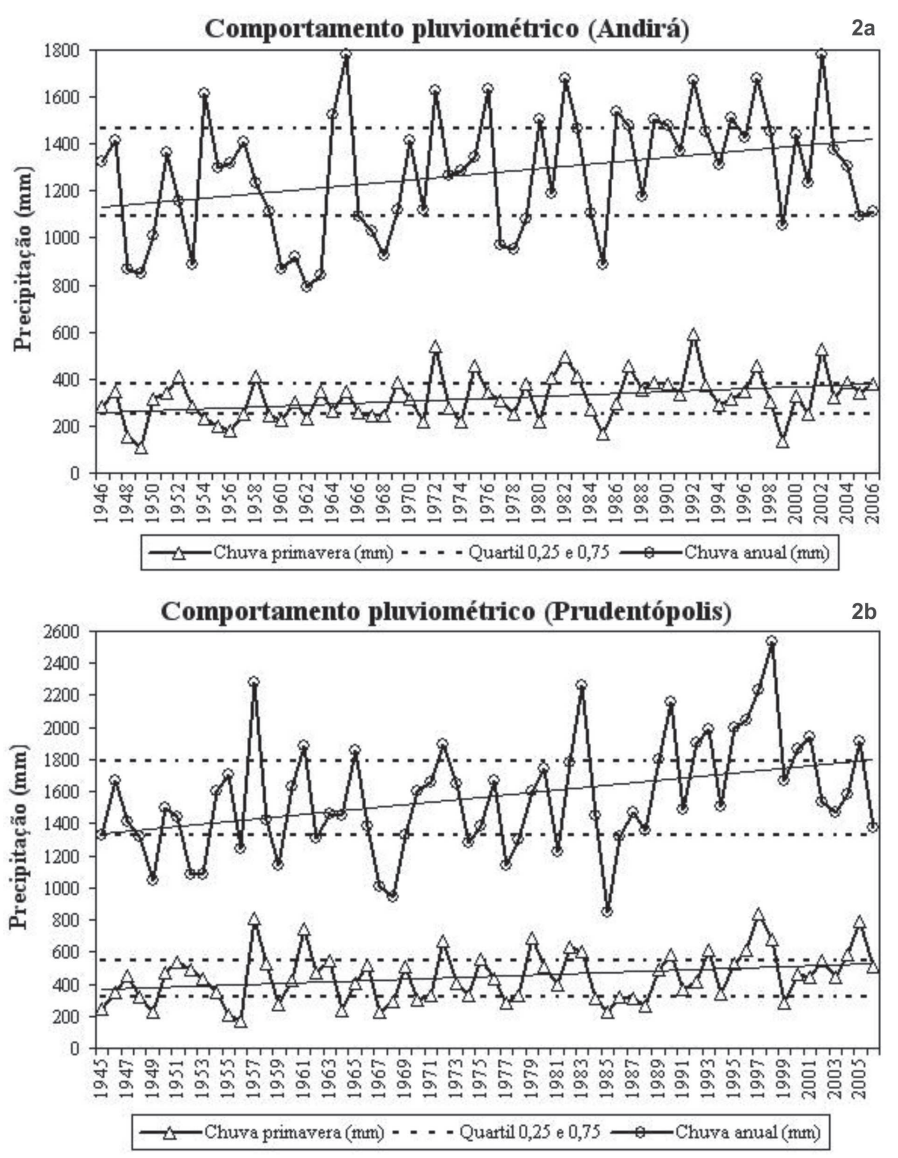

Figura 2. Comportamento da chuva anual e na primavera na estação hidrológica de Andirá (2a) e Prudentópolis (2b) delimitado pelos quartis 0,25 e 075 e linhas de tendência.

Rev. Ceres, Viçosa, v. 58, n.5, p. 593-602, set/out, 2011 
to nos extremos diários de chuva, em todos os períodos sazonais, mas o primeiro não apresenta nenhuma tendência de aumento nas quantidades sazonais, assim como não há uma associação de sinais em outros municípios, para determinados períodos sazonais. Assim, esses resultados para o Paraná contrariam, em parte, os obtidos pelo IPCC (2007) e Haylock et al. (2006). Neste último estudo, os cientistas encontraram praticamente o mesmo padrão nas tendências de eventos extremos e na quantidade de chuva anual, para a América do Sul, durante as últimas décadas. Isto ratifica a importância da análise da variabilidade climática em escalas regionais. Na contrapartida, citam-se alguns municípios onde o aumento nas quantidades de chuva pode ser explicado pelo aumento no número de ocorrências de extremos diários, como em Antonina (inverno e primavera), em Cândido de Abreu (outono), Prudentópolis (verão e primavera), Morretes e Quitandinha (inverno), Ponta Grossa e Tibaji (primavera).

As tendências, quanto ao número de dias secos (inferior a $1 \mathrm{~mm}$ ), não apresentam, rigorosamente, uma associação inversa ao aumento das quantidades acumuladas de chuva. Numa análise geral, apesar da tendência predominante de diminuição no número de dias secos (Tabela 6a), os resultados sugerem uma associação com questões de microclima ou de pequenos erros de leitura diários, que acabam não sendo perceptíveis em análises estatísticas como o Teste de sequência (Run Test). Para Santa Catarina, Camargo et al. (2006) encontraram tendência de aumento, ao longo dos anos, da chuva anual e da variabilidade. Porém, apesar desta tendência, o número de dias consecutivos sem chuva também tem aumentado nos últimos anos, o que significa aumento na ocorrência de eventos extremos.

$\mathrm{Na}$ análise de diferentes tipos de veranicos, percebese, inicialmente, que os veranicos, com quantidades acumuladas inferiores a $1 \mathrm{~mm}$ em 5 dias, apresentam tendência de diminuição, predominantemente no outono e na primavera (Tabela 6b). Chama a atenção o grande número de estações hidrológicas com esta tendência durante o outono, tendo em vista o pequeno número de tendência significativa para as quantidades acumuladas e eventos extremos diários. Isto sugere que, durante o outono, houve aumento no número de dias chuvosos com pequenas quantidades, o que é ratificado pela diminuição do número de dias secos (Tabela 6a). Para a primavera, e anualmente, os resultados são coerentes, já que, nestes períodos, o aumento nas quantidades acumuladas sugere estar refletindo-se na diminuição deste tipo de veranico.

Já para os veranicos com duração de 5 a 10 dias, com quantidade acumulada inferior a $10 \mathrm{~mm}$ (Tabela $6 \mathrm{c}$ e $6 \mathrm{~d}$, respectivamente), o número de tendências significativas (predominantemente negativas) foi bem menor, porém, mais perceptível na primavera e na escala anual.

Seguindo a tendência de aumento na quantidade de chuvas, observado em grande parte do Paraná, as informações da Tabela 7 mostram que a média dos anos mais recentes (1991 a 2006) é significativamente maior que a média do período considerado, atualmente, como "normal climatológica' (1961 a 1990). Porém, praticamente não há resultados suficientes para se afirmar que o grau de dispersão (variabilidade) da chuva anual entre estes dois períodos seja significativamente diferente.

Tabela 5. Tendência da intensidade dos extremos diários da chuva sazonal em alguns municípios do Paraná

\begin{tabular}{lcccc}
\hline Municípios & Verão & Outono & Inverno & Primavera \\
\hline Adrianópolis & ++ & ++ & ++ & ++ \\
Andira & $\mathrm{ns}$ & $\mathrm{ns}$ & $\mathrm{ns}$ & $\mathrm{ns}$ \\
Antonina & $\mathrm{ns}$ & $\mathrm{ns}$ & ++ & ++ \\
Cândido de Abreu & $\mathrm{ns}$ & ++ & $\mathrm{ns}$ & $\mathrm{ns}$ \\
Curitiba & $\mathrm{ns}$ & $\mathrm{ns}$ & $\mathrm{ns}$ & $\mathrm{ns}$ \\
Foz do Iguaçu & - & - & $\mathrm{ns}$ & $\mathrm{ns}$ \\
Guarapuava & $\mathrm{ns}$ & $\mathrm{ns}$ & $\mathrm{ns}$ & $\mathrm{ns}$ \\
Ivaiporã & -- & $\mathrm{ns}$ & + & ++ \\
Morretes & $\mathrm{ns}$ & $\mathrm{ns}$ & $\mathrm{ns}$ & $\mathrm{ns}$ \\
Pato Branco & $\mathrm{ns}$ & $\mathrm{ns}$ & ++ \\
Ponta Grossa & $\mathrm{ns}$ & $\mathrm{ns}$ & $\mathrm{ns}$ & $\mathrm{ns}$ \\
Porto Vitória & $\mathrm{ns}$ & $\mathrm{ns}$ & $\mathrm{ns}$ \\
Prudentópolis & ++ & $\mathrm{ns}$ & ++ & + \\
Quedas do Iguaçu & $\mathrm{ns}$ & $\mathrm{ns}$ & ++ & $\mathrm{ns}$ \\
Quitandinha & + & $\mathrm{ns}$ & + & $\mathrm{ns}$ \\
Rio Negro & ++ & + & $\mathrm{ns}$ & $\mathrm{ns}$ \\
Santa Isabel do Ivaí & ++ & $\mathrm{ns}$ & $\mathrm{ns}$ \\
Tibaji & $\mathrm{ns}$ & $\mathrm{ns}$ & $\mathrm{ns}$ \\
Londrina & $\mathrm{ns}$ & $\mathrm{ns}$ & $\mathrm{ns}$ \\
Palmas & $\mathrm{ns}$ & ++ & & \\
Mariluz & $\mathrm{ns}$ & & & ++ \\
\hline
\end{tabular}

ns=não significativo; ++ e - Significativo a $5 \% ;+$ e - Significativo a $10 \%$. 
Tabela 6. Tendências sazonal e anual de dias secos (6a), com sequência de 5 dias com quantidades totais inferior a $1 \mathrm{~mm}(6 \mathrm{~b})$, com sequência de 5 dias com quantidades totais inferior a $10 \mathrm{~mm}(6 \mathrm{c})$ e com sequência de 10 dias com quantidades totais inferior a 10mm $(6 \mathrm{~d})$

\begin{tabular}{|c|c|c|c|c|c|}
\hline \multirow{2}{*}{$\begin{array}{l}6 \mathrm{a} \\
\text { Municípios }\end{array}$} & \multicolumn{5}{|c|}{ Número de dias secos (chuva < 1mm) } \\
\hline & Verão & Outono & Inverno & Primavera & Anual \\
\hline Adrianópolis & ns & + & ++ & ++ & ++ \\
\hline Andira & -- & -- & ns & -- & -- \\
\hline Antonina & ns & ns & ns & ns & ns \\
\hline Cândido de Abreu & ns & ns & ns & ns & ns \\
\hline Curitiba & ns & ns & ns & ns & ++ \\
\hline Foz do Iguaçu & ns & -- & ns & -- & ns \\
\hline Guarapuava & - & -- & ns & -- & -- \\
\hline Ivaiporã & -- & -- & ns & -- & -- \\
\hline Morretes & -- & - & ns & -- & -- \\
\hline Pato Branco & ns & $\mathrm{ns}$ & ns & $\mathrm{ns}$ & ns \\
\hline Ponta Grossa & - & ns & ns & -- & -- \\
\hline Porto Vitória & ns & ns & ns & ns & ns \\
\hline Prudentópolis & ns & ns & ns & ns & ns \\
\hline Quedas do Iguaçu & -- & -- & -- & -- & -- \\
\hline Quitandinha & ns & ns & ns & - & ns \\
\hline Rio Negro & ns & ns & ns & ns & -- \\
\hline Santa Isabel do Ivaí & ++ & + & ++ & ++ & ++ \\
\hline Tibaji & -- & -- & ns & -- & -- \\
\hline Londrina & ns & ns & ns & ns & ns \\
\hline Palmas & ns & ns & ns & ns & ns \\
\hline Mariluz & ns & ns & ++ & ns & ++ \\
\hline
\end{tabular}

\begin{tabular}{|c|c|c|c|c|c|}
\hline \multirow{2}{*}{$\begin{array}{l}\text { 6b } \\
\text { Municípios }\end{array}$} & \multicolumn{5}{|c|}{ Sequência de 5 dias com totais $<1 \mathrm{~mm}$} \\
\hline & Verão & Outono & Inverno & Primavera & Anual \\
\hline Adrianópolis & $\mathrm{ns}$ & $\mathrm{ns}$ & $\mathrm{ns}$ & ++ & + \\
\hline Andira & -- & -- & ns & -- & -- \\
\hline Antonina & ns & ns & ns & ns & ns \\
\hline Cândido de Abreu & ns & ns & ns & ns & ns \\
\hline Curitiba & ns & ns & ns & ns & + \\
\hline Foz do Iguaçu & ns & -- & ns & - & -- \\
\hline Guarapuava & ns & -- & ns & - & -- \\
\hline Ivaiporã & - & -- & ns & -- & -- \\
\hline Morretes & ns & - & -- & -- & -- \\
\hline Pato Branco & -- & -- & ns & -- & -- \\
\hline Ponta Grossa & ns & ns & ns & - & -- \\
\hline Porto Vitória & ns & ns & ns & ns & ns \\
\hline Prudentópolis & ns & ns & ns & ns & ns \\
\hline Quedas do Iguaçu & -- & -- & ns & -- & -- \\
\hline Quitandinha & ns & ns & ns & ns & - \\
\hline Rio Negro & ns & - & ns & ns & ns \\
\hline Santa Isabel do Ivaí & ++ & ns & ++ & ns & ns \\
\hline Tibaji & -- & -- & ns & -- & -- \\
\hline Londrina & ns & ns & ns & ns & ns \\
\hline Palmas & ns & ns & ns & ns & ns \\
\hline Mariluz & ns & ns & - & ns & ns \\
\hline
\end{tabular}

\begin{tabular}{|c|c|c|c|c|c|}
\hline \multirow{2}{*}{$\begin{array}{l}\text { 6c } \\
\text { Municípios }\end{array}$} & \multicolumn{5}{|c|}{ Sequência de 5 dias com totais $<10 \mathrm{~mm}$} \\
\hline & Verão & Outono & Inverno & Primavera & Anual \\
\hline Adrianópolis & ns & ++ & $\mathrm{ns}$ & ns & ++ \\
\hline Andira & ns & ns & ns & -- & -- \\
\hline Antonina & ns & ns & ns & - & ns \\
\hline Cândido de Abreu & ns & - & ns & ns & -- \\
\hline Curitiba & ns & ns & ns & ns & ns \\
\hline Foz do Iguaçu & ns & ns & ns & ns & ns \\
\hline Guarapuava & - & ns & ns & -- & - \\
\hline Ivaiporã & ns & ns & ns & ns & ns \\
\hline Morretes & ns & $\mathrm{ns}$ & ns & -- & -- \\
\hline Pato Branco & ns & ns & ns & -- & $\mathrm{ns}$ \\
\hline Ponta Grossa & ns & ns & ns & ns & ns \\
\hline Porto Vitória & ns & ns & ns & ns & ns \\
\hline Prudentópolis & -- & ns & ns & ns & -- \\
\hline Quedas do Iguaçu & ns & ns & ns & ns & ns \\
\hline Quitandinha & ns & ns & ns & ns & ns \\
\hline Rio Negro & - & - & ns & ns & -- \\
\hline Santa Isabel do Ivaí & ns & ns & ns & -- & ns \\
\hline Tibaji & ns & ns & ns & - & ns \\
\hline Londrina & ns & ns & ns & ns & ns \\
\hline Palmas & ns & ns & ++ & ns & ns \\
\hline Mariluz & ns & ns & ns & ns & ns \\
\hline
\end{tabular}

Rev. Ceres, Viçosa, v. 58, n.5, p. 593-602, set/out, 2011 


\begin{tabular}{|c|c|c|c|c|c|}
\hline \multirow{2}{*}{$\begin{array}{l}\text { 6d } \\
\text { Municípios }\end{array}$} & \multicolumn{5}{|c|}{ Sequência de 10 dias com totais $<10 \mathrm{~mm}$} \\
\hline & Verão & Outono & Inverno & Primavera & Anual \\
\hline Adrianópolis & + & ns & ns & ns & ++ \\
\hline Andira & ns & ns & ns & -- & -- \\
\hline Antonina & ns & ns & - & ns & ns \\
\hline Cândido de abreu & ns & ns & ns & ns & ns \\
\hline Curitiba & ns & ns & ns & ns & ns \\
\hline Foz do Iguaçu & ns & - & ns & ns & ns \\
\hline Guarapuava & ns & ns & ns & ns & ns \\
\hline Ivaiporã & ns & ns & ns & ns & ns \\
\hline Morretes & ns & $\mathrm{ns}$ & - & - & -- \\
\hline Pato Branco & ns & ns & ns & ns & ns \\
\hline Ponta Grossa & ns & ns & ns & ns & ns \\
\hline Porto Vitória & ns & $\mathrm{ns}$ & ns & ns & ns \\
\hline Prudentópolis & -- & ns & ns & ns & -- \\
\hline Quedas do Iguaçu & ns & ns & ns & ns & ns \\
\hline Quitandinha & ns & ns & ns & ns & ns \\
\hline Rio Negro & - & ns & ns & ns & -- \\
\hline Santa Isabel do Ivaí & ns & ns & ns & ns & ns \\
\hline Tibaji & - & ns & ns & ns & - \\
\hline Londrina & ns & ns & ns & ns & ns \\
\hline Palmas & $\mathrm{ns}$ & $\mathrm{ns}$ & $\mathrm{ns}$ & ns & $\mathrm{ns}$ \\
\hline Mariluz & ns & ns & ns & ns & ns \\
\hline
\end{tabular}

ns=não significativo; ++ e - Significativo a $5 \% ;+$ e - Significativo a $10 \%$.

Tabela 7. Teste de igualdade da média e variância da chuva anual entre os períodos 1961 a 1990 e 1991 a 2006

\begin{tabular}{lcc}
\hline Municípios & Média & Variância \\
\hline Adrianópolis & $\mathrm{ns}$ & $\mathrm{ns}$ \\
Andira & - & $\mathrm{ns}$ \\
Antonina & - & $\mathrm{ns}$ \\
Cândido de Abreu & $\mathrm{ns}$ & $\mathrm{ns}$ \\
Curitiba & $\mathrm{ns}$ & $\mathrm{ns}$ \\
Foz do Iguaçu & - & + \\
Guarapuava & - & $\mathrm{ns}$ \\
Ivaiporã & $\mathrm{ns}$ & $\mathrm{ns}$ \\
Morretes & - & $\mathrm{ns}$ \\
Pato Branco & $\mathrm{ns}$ & $\mathrm{ns}$ \\
Ponta Grossa & $\mathrm{ns}$ & $\mathrm{ns}$ \\
Porto Vitória & $\mathrm{ns}$ & $\mathrm{ns}$ \\
Prudentópolis & - & $\mathrm{ns}$ \\
Quedas do Iguaçu & $\mathrm{ns}$ & $\mathrm{ns}$ \\
Quitandinha & $\mathrm{ns}$ & $\mathrm{ns}$ \\
Rio Negro & - & $\mathrm{ns}$ \\
Santa Isabel do Ivaí & $\mathrm{ns}$ & $\mathrm{ns}$ \\
Tibaji & $\mathrm{ns}$ & $\mathrm{ns}$ \\
Londrina & $\mathrm{ns}$ & $\mathrm{ns}$ \\
Palmas & $\mathrm{ns}$ & $\mathrm{ns}$ \\
Mariluz & & $\mathrm{ns}$ \\
\hline & &
\end{tabular}

ns = não significativo; — e - Média/variância de 1961-90 menor que a média/variância de 1991-06 a 5 e 10\%, respectivamente; ++ e + Média/variância de 1961-90 maior que a média/variância de 1991-06 a 5 e $10 \%$, respectivamente.

\section{CONCLUSÕES}

Não houve identificação de algum ciclo climático nas quantidades de chuvas sazonal e anual.

Houve aumento na quantidade de chuvas desde meados do século XX, principalmente durante a primavera e na metade leste do Paraná.
O número de dias com eventos extremos de chuva mostra tendência de aumento. Porém, os municípios e períodos sazonais com esta característica não seguem rigorosamente as tendências apresentadas na quantidade de chuva.

A diminuição da ocorrência de veranicos na primavera, com duração de cinco dias, é inversamente proporcional ao aumento na quantidade de chuva.

\section{REFERÊNCIAS}

Alexandersson H (1986) A homogeneity test applied to precipitation data. Journal of Climatology, 6:661-675.

Alexandersson H, Moberg A (1997) Homogenization of Swedish temperature data. Part 1: homogenity test for linear trends. International Journal of Climatology, 17:25-34.

Back AJ (2001) Aplicação de análise estatística para identificação de tendências climáticas. Pesquisa Agropecuária Brasileira, 36:717-726.

Camargo CG, Braga HJ \& Alves RCM (2006) Mudanças climáticas atuais e seus impactos no Estado de Santa Catarina. Revista Agropecuária Catarinense, 19:31-35.

Cavalcanti IFA \& Kousky VE (2009) Frentes frias sobre o Brasil. In: Cavalcanti IFA, Ferreira NJ, Justi da Silva MGA \& Silva Dias MAF (Eds.) Tempo e clima no Brasil. São Paulo, Oficina de Textos. p.135-148.

Diaz AF, Studzinski CD \& Mechoso CR (1998) Relationship between precipitation anomalies in Uruguay and Southern Brazil and sea surface temperature in the Pacific and Atlantic oceans. Journal of Climate, 11:251-271.

Diniz FA, Nascimento FA, Sousa Silva VB \& Figueiredo AAD (1998) Relação do El Niño 1997/98 com a estiagem no norte e nordeste de Minas Gerais período: outubro/1997 a abril/1998. In: $10^{\circ}$ Congresso Brasileiro de Meteorologia, Brasília. Anais, SBMet. CD-ROM.

Feidas H, Noulopoulou C, Makrogiannis T \& Bora-Senta E (2007) Trend analysis of precipitation time series in Greece and their relationship with circulation using surface and satellite data: 1951-2001. Theoretical and Applied Climatology, 87:155-177. 
Grimm AM (2002) The El Niño impact on the summer monsoon in Brazil: Regional processes versus remote influences. Journal of Climate, 16:263-280.

Haylock MR, Peterson T, Abreu de Sousa JR, Alves LM, Ambrizzi T, Anunciação YMT, Baez J, Barbosa de Brito JI, Barros VR, Berlato MA, Bidegain M, Coronel G, Corradi V, Garcia VJ, Grimm AM, Jaildo dos Anjos R, Karoly D, Marengo JA, Marino MB, Meira PR, Miranda GC, Molion L, Moncunill DF, Nechet D, Ontaneda G, Quintana J, Ramirez E, Rebello E, Rusticucci M, Santos JL, Trebejo I \& Vincent L (2006) Trends in total and extreme South American temperature 1960-2000 and links with sea surface temperature. Journal of Climate, 19:14901512.

IPCC. 2007. Summary for policymakers. In: Solomon, S.; Qin, D.; Manning, M.; Chen, Z.; Marquis, M.; Averyt, K.B.; Tignor, M.; Miller, H.L. (eds) Climate change 2007: the physical science basis. Contribution of Working Group I to the Fourth Assessment Report of the Intergovernmental Panel on Climate Change. Cambridge University Press, Cambridge.

Kendall M (1938) A new measure of rank correlation. Biometrika, $30: 81-89$.
Kunkel K (2003) North American trends in extreme precipitation. Nature Hazards, 29:291-305.

Marengo JA (2004) Interdecadal variability and trends of rainfall across the Amazon basin. Theoretical and Applied Climatology, doi: 10.1007/s00704-004-0045-8

Obregón G \& Nobre CA (2003) Rainfall trends in Brazil. Bulletin American Meteorological Society, 84:1008-1009.

Obregón G \& Marengo JA (2007) Caracterização do clima do século XX no Brasil: Tendências de chuvas e temperaturas médias e extremas. Relatório no 2. Mudanças climáticas Globais e efeitos sobre a biodiversidade. Brasília, Ministério do Meio Ambiente. 91p.

Re M \& Barros VR (2009) Extreme rainfalls in SE South America. Climatic Change, 96:119-136.

Silva Dias MAF (Ed.) (2009) As chuvas de novembro de 2008 em Santa Catarina: um estudo de caso visando à melhoria do monitoramento e da previsão de eventos extremos. Nota técnica. São José dos Campos, INPE. 67p.

Thom, HCS (1966) Some methods of climatological analysis. Geneva: World Meteorological Organization, 53p. (Technical Note, $n^{\circ} 81$ ). 